\title{
System for Automatic Processing and Analysis of MRI/fMRI Data on the Kurchatov Institute Supercomputer
}

\author{
Irina M. Enyagina ${ }^{1, \star}$, Andrey N. Polyakov ${ }^{1}$, Alexey A. Poyda ${ }^{1}$, and Vadim L. Ushakov ${ }^{1}$ \\ ${ }^{1}$ Department of Neurocognitive Sciences, National Research Center «Kurchatov Institute», Moscow, Russia
}

\begin{abstract}
This paper presents the Computer Model of the System for Automatic Processing and Analysis of MRI/fMRI tomography data, obtained at the Kurchatov Institute Resource Center "Cognimed". The System is based on the "Digital Lab" IT-Platform, involving the Kurchatov Institute Supercomputer Cluster HPC4, which allows speeding up the processing of data for groups (2-350 subjects) by parallelization of computations on the supercomputer nodes ( 1 subject -1 node). The proposed System allows scientists to remotely use the installed on the supercomputer specialized software to process and analyze MRI/fMRI data; organizes a unified data storage; permits the work with data by web a interface. The System also enables the use of program modules developed by KI researchers which implement mathematical methods to improve data analysis results. As an example of the realization of this Computer Model, the Module "MRI FS" is presented that provides automatic processing and analysis of MRI data using the open specialized software FreeSurfer v.6.0.
\end{abstract}

\section{Introduction}

Two main subject matters in the highly actual study of the human brain are the brain anatomic structure and the functional neural networks architecture. Results of these researches are used in machine intelligence, bioinformatics and in clinical practice. The development of MRI and fMRI tomography methods offered many new opportunities for human brain research. MRI (magnetic resonance imaging) is used for the visualization of the brain anatomical structure. fMRI (functional magnetic resonance imaging) is used for measuring the functional activity of the brain [1]. As the number of experiments increases, the need arises to improve and automate the processing and analysis of MRI and fMRI data. 1) An MRI/fMRI experiment for one subject takes 15-40 minutes, while the processing of the collected experimental data can take 24 hours or more, so the processing of data for one day of tomographic experiments can take 30 or more days of calculations on one PC. Therefore, parallelization of computational processes is necessary. It is especially relevant for group analysis, when it is necessary to process the data of a large number of subjects at the same time (usually 20-200 subjects). 2) Tomographic data requires a lot of space, which can be a problem for PC, so an important task is the creation of a unified centralized data storage containing information about all stages of data processing and analysis. This will secure the safety of the data and will allow the possibility of fast access to it, together with the reuse of the same data for various studies. 3) There are many different

^e-mail: irina_enyagina@mail.ru 
tools for the analysis of MRI and fMRI data, but it is not always possible to install all of them on one PC. Therefrom the need to organize a data processing and analysis center that provides a wide collection of remotely placed specialized tools. 4) The development of more user-friendly interfaces, like a web interface, is desired to allow the processing and analysis of MRI/fMRI data by persons without specialized IT skills. 5) The mathematical methods for analyzing MRI / fMRI data need to be improved [2,3]. Scientists at the Kurchatov Institute are currently working on the creation of more advanced data processing mathematical methods for discovering functional brain networks and on the development of program modules that would provide the use of these methods in practice [4]. Therefore, there is the task of integrating such program modules into a general model of processing and analyzing MRI/fMRI data. To solve these tasks, the Computer Model of the System for Automatic Processing and Analysis of MRI/fMRI experimental data was developed based on the "Digital Lab" IT-Platform [5], involving the Kurchatov Institute Supercomputer Cluster HPC4 [6].

\section{Data work flows in MRI/fMRI experiments}

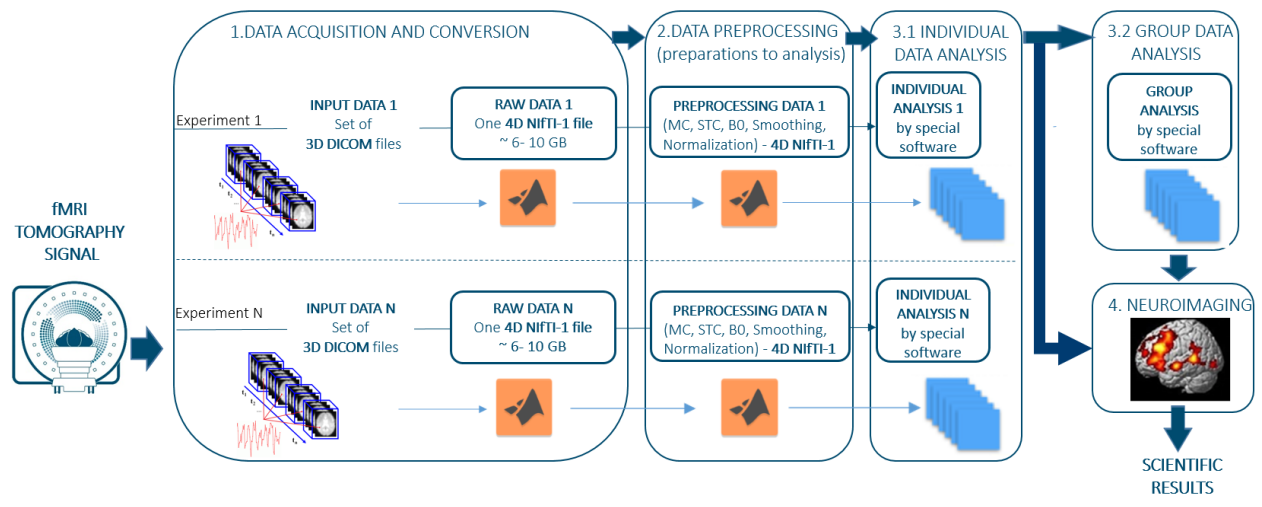

Figure 1. (Color online) fMRI experiments data work flow.

Data work flows in MRI/fMRI experiments consist of the following stages (figure 1): 1. DATA ACQUISITION AND CONVERSION. The MRI / fMRI tomographic signal is recorded on the operator's PC as a set of DICOM files (2D DICOM for MRI, 3D DICOM for fMRI), and this is "Input data". Then the DICOM file set is converted to a single NIfTI-1 file (3D NIfTI-1 50 MB for MRI, 4D NIfTI$16-14$ GB for fMRI), and this is "Raw Data". 2. DATA PREPROCESSING. Preparing data for analysis, removing unnecessary information from a NIfTI-1 file. 3. DATA ANALYSIS. This stage takes the NIfTI-1 file as input and produces files (in various formats) with data analysis results. 3.1. "Individual Data Analysis" (for one subject). 3.2 (if necessary) "Group Data Analysis" (usually for groups of up to 200 subjects). 4. NEUROIMAGING. Visualization of data analysis results by means of special software tools.

\section{The system for automatic processing and analysis of MRI/fMRI data}

The System has the following architecture (figure 2): (1) Data Management Module: Organizes the exchange of data between System's Modules. (2) Data Converting Module: conversion of tomography data from DICOM to NIfTI-1 format. (3) Data Storage: Unified storage of the data and of the results of 
their processing and analysis. (4) Computing Module: Data processing and analysis using specialized software. Parallelization of calculations. (5) Analytics and Statistics Module: Generation of analytical and statistical reports. (6) Data Visualization Module: Creation of data neuroimaging models. (7) Web Interface: Remote access to the System for external devices.

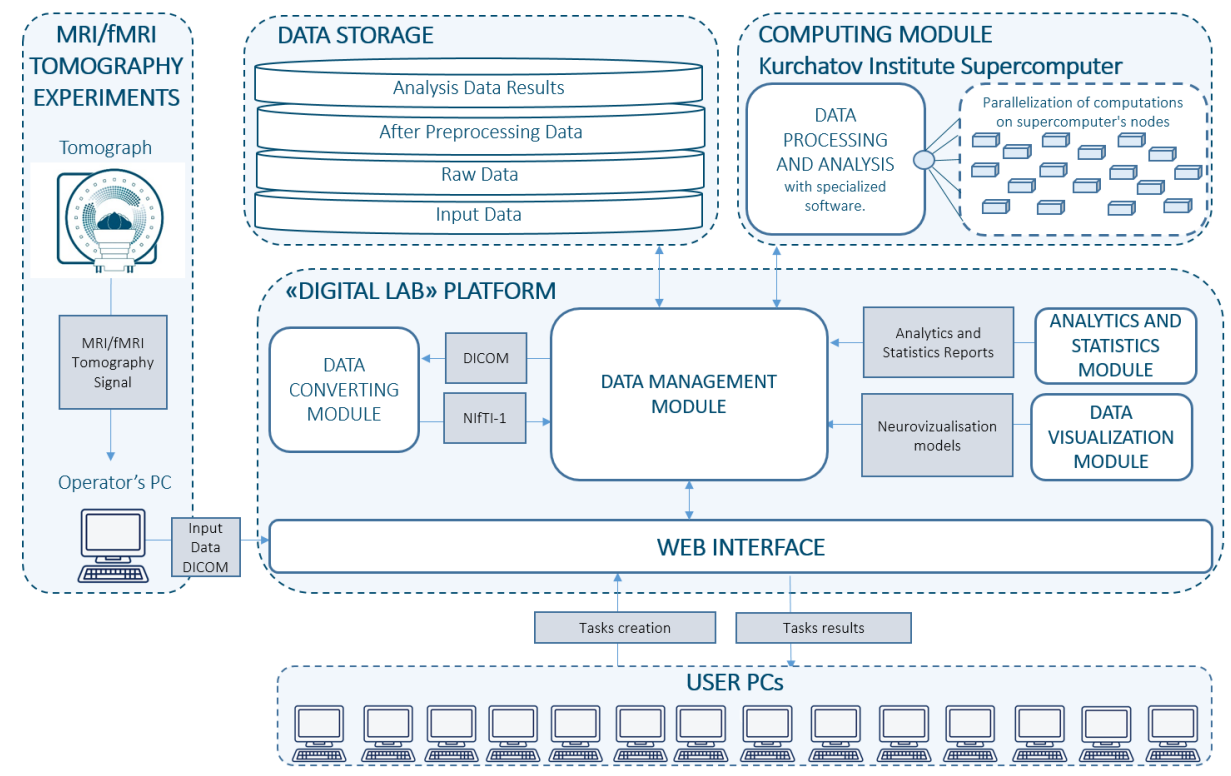

Figure 2. (Color online) Computer model of the System for Automatic Processing and Analysis of MRI/fMRI data.

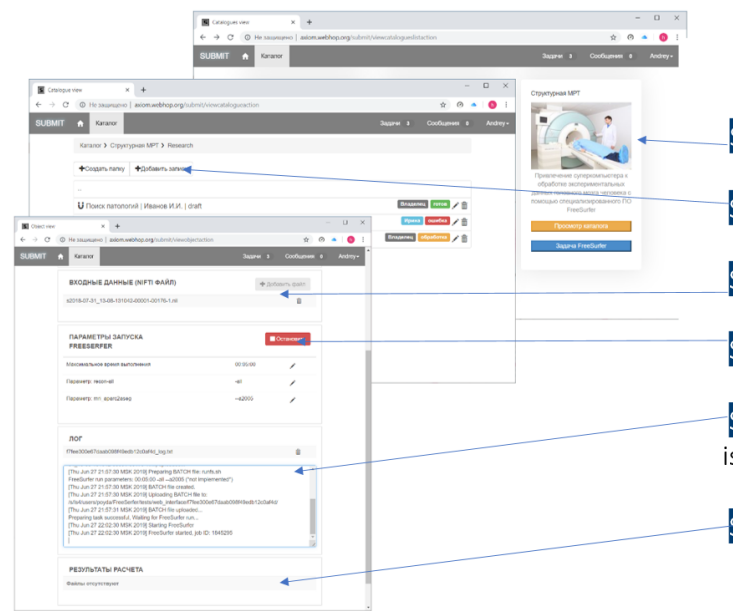

MODULE «MRI FS»

Algorithm for users

Step 1 Log in to the portal

Step 2 Create new subject record

Step 3 Attach MRI data

Step 4 Start task by pressing «Start» button

Step 5 Waiting for results (an interactive journal is provided for monitoring the status of work)

Step 6 Download .rar file with results

Figure 3. (Color online) Module "MRI FS". Algorithm for users.

Based on this Computer Model, the Module "MRI FS" was created and implemented at the Kurchatov Institute. This Module realized the whole workflow, proposed in the Computer Model, for the 
processing and analysis of MRI data (obtained on the Tomograph Siemens Verio Magnetom 3T at the KI Resource Center "Cognimed") using open specialized software (FreeSurfer v.6.0 [7], installed on the KI Supercomputer (Cluster HPC4 [6]). Figure 3 shows the algorithm for users working with the module through the web interface. One of the features of the Module "MRI FS" is the automatic generation of scripts that run data processing and analysis tasks for each subject of the group. An important task was to provide remote access, since the experimental equipment (Tomograph) and computing resources (Supercomputer) are located in different buildings of the Kurchatov Institute. The use of the Module "MRI FS" allows the simplification and speed up of the data processing. Now, it is enough for neuroscientists to download raw data (DICOM) via the web interface, and within 24 hours to get the result of data processing and analysis. For example, using the Module "MRI FS" allows to process data for a group of 100 subjects during a 24 hours interval, whereas earlier it could take about three months.

\section{Conclusion}

We have created the Computer Model of the System for Automatic Processing and Analysis of MRI/fMRI data which allows organizing the interaction between the Resource Center "Cognimed", the Computer Center Complex for Simulation and Data Processing and the scientists at the Kurchatov Institute (Moscow). We accelerated data processing for groups of 2-350 subjects by parallelizing computations on the supercomputer's nodes ( 1 subject -1 node). We are also working on the task of parallelizing computations on the supercomputer's nodes for one subject (1 subject - several nodes). The Module "MRI FS" which covers the whole proposed Computer Model, has been implemented for automatic processing and analysis of MRI data using the open specialized software FreeSurfer [7], with remote users access by the web interface. We are currently working on the creation of new modules for processing and analysis of MRI / fMRI data, based on the proposed Computer Model.

\section{Acknowledgements}

The work was supported by the NRC «Kurchatov Institute» (№ 1359), project "Creation of a distributed modular research and development platform 'Digital laboratory" "; by the RFBR research project № 18-29-23020 mk "Investigation of functional architecture of the human brain resting state networks as the basic model of energy-efficient information processes of consciousness"; and by the RFBR research project № 17-29-02518 ofi-m "Basic cognitive-effective structures of the human brain in norm and in patients with schizophrenia".

\section{References}

[1] R.A. Poldrack et al, Handbook of Functional MRI Data Analysis (Cambridge University Press; 2011)

[2] K. Görgenab, M.N. Hebart, et al, NeuroImage 180, Part A, 19-30 (2018)

[3] V.A. Orlov, V.L. Ushakov, et al, in Biologically Inspired Cognitive Architectures 2019, Advances in Intelligent Systems and Computing 948, 400-404 (2019)

[4] I.M. Enyagina, A.A. Poyda, et al, International Congress "Neuroscience for Medicine and Psychology", https://doi .org/10.29003/m384. sudak.ns2019-15/170-171 (2019)

[5] A.N. Polyakov, D.S. Kokovin, et al, Procedia Computer Science 119, 59-64 (2017)

[6] Kurchatov Institute Supercomputer Cluster HPC4 description [in Russian] http://www . computing.nrcki.ru/pages/main/12530/12546/index.shtml

[7] FreeSurfer Software Suite, https://surfer.nmr.mgh.harvard.edu/ 